\title{
$s$-Wave Paired Electron and Hole Composite Fermion Trial State for Quantum Hall Bilayers with $\nu=1$
}

\author{
Glenn Wagner®, ${ }^{1,2}$ Dung X. Nguyen, ${ }^{3}$ Steven H. Simon, ${ }^{1}$ and Bertrand I. Halperin $\odot^{4}$ \\ ${ }^{1}$ Rudolf Peierls Centre for Theoretical Physics, University of Oxford, Oxford OX1 3PU, United Kingdom \\ ${ }^{2}$ Kavli Institute for Theoretical Physics, University of California, Santa Barbara, California 93106, USA \\ ${ }^{3}$ Brown Theoretical Physics Center, Brown University, Providence, Rhode Island 02912, USA \\ ${ }^{4}$ Department of Physics, Harvard University, Cambridge, Massachusetts 02138, USA
}

(Received 11 June 2021; accepted 12 November 2021; published 10 December 2021)

\begin{abstract}
We introduce a new variational wave function for a quantum Hall bilayer at total filling $\nu_{T}=1$, which is based on $s$-wave BCS pairing between electron composite fermions in one layer and hole composite fermions in the other. In addition, we reexamine a trial wave function based on $p$-wave BCS pairing between electron composite fermions in both layers. We compute the overlap of the optimized trial functions with the ground state from exact diagonalization calculations of up to 14 electrons in a spherical geometry, and we find excellent agreement over the entire range of values of the ratio between the layer separation and the magnetic length. The $s$-wave trial wave function naturally allows for charge imbalance between the layers and provides important insights into how the physics at large interlayer separations crosses over to that at small separations in a fashion analogous to the BEC-BCS crossover.
\end{abstract}

DOI: 10.1103/PhysRevLett.127.246803

Introduction.-The bilayer quantum Hall system at total filling fraction $\nu=1$ has been the subject of considerable theoretical and experimental interest for more than two decades [1,2]. The $\nu=1$ bilayer quantum Hall system consists of two two-dimensional electron systems separated by a distance $d$. A magnetic field $B$ perpendicular to the layers is applied such that $\nu=n \phi_{0} / B=1$, with $n=n_{\uparrow}+$ $n_{\downarrow}$ the total electron density and $\phi_{0}=2 \pi \hbar / e$ the flux quantum ( $\uparrow$ and $\downarrow$ refer to the two different layers). The competition between inter- and intralayer Coulomb interactions makes this system both interesting and challenging.

We assume here that all electrons are confined to the lowest Landau level and are fully spin polarized. At small interlayer distances $d$ compared to the magnetic length $\ell_{B}=\sqrt{\hbar / e B}$, the ground state can be described as an exciton condensate: the electrons in one layer form tightly bound states with the holes in the opposite layer $[1,3]$. In contrast, at infinite interlayer distances, the two layers decouple completely such that each layer forms an independent composite fermion (CF) liquid [4-6]. Since these two limits are described in terms of different quasiparticles, understanding how they are connected is a difficult problem. There has been an enormous amount of theoretical work attempting to address this question [2,7-39].

In a recent paper by one of the current authors and one with other collaborators [40,41], a new approach to this crossover was proposed: $s$-wave BCS pairing of electron CFs (eCFs, two flux quanta bound to an electron) in one layer with hole-CFs (hCFs, two flux quanta bound to a hole) in the other layer. This approach qualitatively appeals in that it describes the correct types of quasiparticles both for small $d$ (excitons) and large $d$ (CFs). Further, it naturally allows a description in the case of imbalanced layers where $n_{\uparrow} \neq n_{\downarrow}$. The purpose of this Letter is to numerically test this proposal.

At small $d / \ell_{B}$, the $\nu=1$ system forms Halperin's (111) state [3]. This state can be viewed as a condensate of interlayer excitons, or equivalently $s$-wave pairing of electrons in one layer with holes in the other. This limit is well described in Hartree-Fock [7] and is a good description even when the density is imbalanced between the layers.

At large $d / \ell_{B}$, the description of the layers is more complicated. For infinite $d / \ell_{B}$, the layers behave as independent quantum Hall states. For the balanced case of $\nu_{\uparrow}=\nu_{\downarrow}=1 / 2$, each layer is well described as a CF Fermi sea in zero effective magnetic field [4-6]. Away from filling $1 / 2$, the CFs see a residual magnetic field.

When the two $\nu=1 / 2$ layers are then weakly coupled together, we expect the $\mathrm{CF}$ liquids to become correlated with each other. A possibility that was considered from very early on is that the two layers form a BCS paired state of eCFs [8-10]. Numerical work with trial wave functions established [26] that the $p$-wave channel is the symmetry channel with the largest gap. In exact diagonalization (ED) studies, the $p$-wave paired state was shown $[25,26]$ to have very high overlaps with the exact ground state for $d \gtrsim \ell_{B}$. In those studies, the overlap rapidly decreased at $d \lesssim \ell_{B}$. However, Ref. [29] argued that the $p$-wave pairing state could be continuously deformed to the exciton condensate without going through a phase transition. 
In recent years, after the initial investigations into the $\nu=1$ bilayer, there has been renewed focus on the issue of particle-hole symmetry in the $\nu=1 / 2 \mathrm{CF}$ Fermi liquid state $[42,43]$. While the single-layer half-filled Landau level is particle-hole symmetric [44], the CF construction $[4,5]$ does not appear to respect this symmetry in any obvious way. This then raises the question as to whether we should view the half-filled Landau level as a Fermi sea of eCFs or as a Fermi sea of hCFs removed from a filled Landau level (we call this an "anti-CF" Fermi sea). While the two descriptions are numerically almost equivalent [45], there may, nonetheless, be advantages to thinking in terms of one or the other.

In this Letter, we examine a new model of the $\nu=1$ bilayer: $s$-wave pairing of the eCFs in one layer with hCFs in the other layer. We show that a trial wave function based on this approach has very high overlaps with the exact ground state at all distances $d / \ell_{B}$. The evolution of the system as a function of $d / \ell_{B}$ is analogous to the BEC-BCS crossover familiar from cold atom gases [46]. At large $d$, we have weakly bound eCF-hCF pairs (BCS limit), whereas at small $d$, we have tightly bound eCF-hCF pairs tending toward the $\mathrm{BEC}$ regime. A nice feature of this approach is that if one considers the Chern-Simons (Halperin-Lee-Read [4]) description of CFs, the s-wave pairing wave function described above exactly recovers the $d \rightarrow 0$ limit as the limit where the $s$-wave pairs have a very small binding radius and the Landau level mixing is neglected $[41,47]$.

This approach also applies just as well to the case of charge imbalance of the layers. If one transfers charge between the layers, the two Fermi seas remain the same size as each other, both growing or shrinking together, so that the pairing is not destroyed. We find that the imbalanced bilayer system still forms an $s$-wave paired state of eCFs and hCFs. We also reexamine a trial wave function based on $p$-wave pairing of eCFs in both layers and find significantly higher overlaps than previous works [25,26] such that the improved overlaps are comparable to the newly introduced $s$-wave trial state.

Details of calculation.-We consider Coulomb interaction $e^{2} / \epsilon r$ for electrons in the same layer (intralayer interaction) and $e^{2} / \epsilon \sqrt{r^{2}+d^{2}}$ for electrons in different layers (interlayer interaction). As mentioned above, we assume that the physical spin of the electrons is completely polarized due to the Zeeman splitting and exchange interaction. We assume zero temperature and no disorder, and we neglect Landau level mixing. We also assume no tunneling between the layers, which is a good approximation of many of the experiments.

We perform ED on the sphere for systems of up to $N=$ 14 electrons, i.e., $N_{\uparrow}=N_{\downarrow}=7$ electrons per layer in the balanced case. More generally, the total number of electrons needs to satisfy $N=N_{\uparrow}+N_{\downarrow}=N_{\phi}+1$, with $N_{\phi}$ the total number of flux quanta passing through the sphere, such that we have total filling $\nu=1$. We particle-hole transform [48] the bottom layer such that we are describing it in terms of $N_{\uparrow}$ hole coordinates. Note in particular that the number of holes in the bottom layer matches the number of electrons in the top layer.

In the top layer, we form eCFs by attaching two Jastrow factors to each electron. In the planar geometry, this would be achieved by multiplying our wave function by $\prod_{i<j}\left(z_{i}-z_{j}\right)^{2}$, where $z_{i}=x_{i}+i y_{i}$ is the complex coordinate of the $i$ th electron at position $\left(x_{i}, y_{i}\right)$. (Adaptation to the spherical geometry is discussed in the Supplemental Material [49].) The effective flux seen by the eCFs is $N_{\phi}^{\text {eff }}=N_{\phi}-2\left(N_{\uparrow}-1\right)$. In the bottom layer, we similarly form hCFs, which see the same effective flux, by attaching two anti-Jastrow factors by multiplying by $\prod_{i<j}\left(w_{i}-w_{j}\right)^{* 2}$, where $w_{i}=X_{i}+i Y_{i}$ is the complex coordinate of a hole at $\left(X_{i}, Y_{i}\right)$ [53]. We then BCS pair the eCFs from the top layer with the hCFs from the bottom layer in the $s$-wave channel. We can write down a variational pairing wave function based on this approach (see the Supplemental Material [49] for details):

$$
\begin{aligned}
\Psi_{\mathrm{BCS}, s} & =\prod_{i<j}\left(z_{i}-z_{j}\right)^{2}\left(w_{i}-w_{j}\right)^{* 2} \operatorname{det}(G) \\
G\left(z_{i}, w_{j}\right) & =\sum_{l, m} g_{l} \phi_{l, m}\left(z_{i}\right) \phi_{l, m}^{*}\left(w_{j}\right),
\end{aligned}
$$

where $\phi_{l, m}$ are the Jain-Kamilla orbitals [54,55] with angular-momentum quantum numbers $l, m$ describing CFs in effective flux $N_{\phi}^{\text {eff }}$. The $g_{l}$ are variational parameters. Because of rotational symmetry, the variational parameters cannot depend on $m$. This trial wave function approach is similar to the BCS $p$-wave pairing approach of Refs. $[25,26]$ except that in that work eCFs are paired with eCFs, whereas here eCFs are paired with hCFs. The assignment of the terms $s$ wave and $p$ wave for the two wave functions can be seen from two properties of the wave function of the sphere (see [49]): (a) the behavior of the wave function as two particles are brought close together: the wave function scales like $r^{\ell}$, where $r$ is the separation of the pair and $\ell$ is the angular momentum; (b) the behavior of the wave function as two particles are exchanged: the wave function picks up a sign $(-1)^{\ell}$ under exchange.

We convert the ED ground state into position space and compute the overlap with the trial state by performing Monte Carlo integration. We use the probability distribution of the (111) state for the importance sampling. The optimal variational parameters that maximize the overlap are found using a dual annealing algorithm [56].

Balanced case.-Let us focus on the case where the two layers are balanced, i.e., $\nu_{\uparrow}=\nu_{\downarrow}=1 / 2$, and the number of electrons per layer on the sphere is $N_{\uparrow}$. We show the overlaps of the $s$-wave variational state with the ED ground state in Fig. 1(a) for $N_{\uparrow}=6$ (see the 

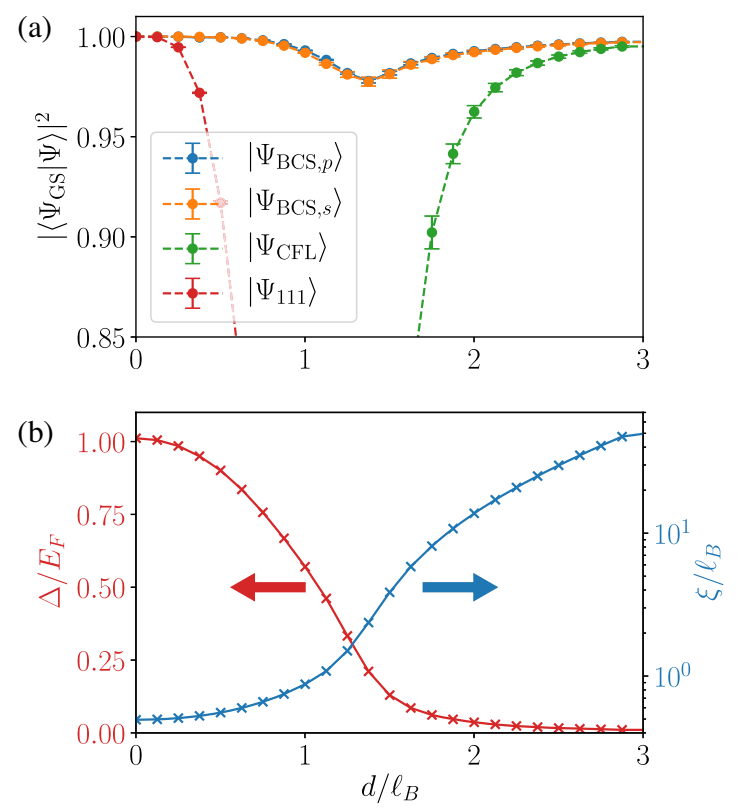

FIG. 1. Exact diagonalization results for a balanced system with $6+6$ electrons on the sphere. (a) We plot the overlap of the trial wave functions with the true ground state $\left|\Psi_{\mathrm{GS}}\right\rangle$ as a function of the interlayer distance $d$. We compare the overlap of our $s$-wave BCS state with the previously proposed $p$-wave BCS state of Refs. [25,26]. The $s$ - and $p$-wave curves are almost indistinguishable on this plot. For both trial wave functions, we include 5 variational parameters. We also show the overlaps with the composite Fermi liquid (CFL) state and the (111) state, which are accurate descriptions of the state in the large and small $d$ limits, respectively. The error bars denote the errors of the Monte Carlo integration. (b) BCS parameters $\Delta / E_{F}$ and $\xi / \ell_{B}$ extracted from the $s$-wave variational wave function from (a). $\Delta$ is the $s$-wave superconducting order parameter, $E_{F}$ is the Fermi energy, and $\xi$ is the coherence length. The evolution of the BCS parameters as a function of the interlayer separation $d$ is consistent with a BEC-BCS crossover.

Supplemental Material [49] for other system sizes). For $N_{\uparrow}=4,5,6,7$, we achieve overlaps squared of better than 0.95 by including $3,4,5,6$ variational parameters, respectively. Given that the Hilbert space dimensions of the $L^{2}=0$ subspace in which the ground state and the trial wave functions lie are $D\left(L^{2}=0\right)=12,38,252,1599$, respectively, the high overlaps obtained are significant. In Fig. 1(a), we also show overlap results for the (111) state and the $p$-wave paired state from Ref. [26], as well as the $\mathrm{CF}$ Fermi liquid state (two uncoupled eCF liquids).

At very large distances, the CF Fermi liquid state is essentially exact for $N_{\uparrow}=2,6,12 \ldots$, where we have enough eCFs to completely fill an integer number of angular momentum shells. For unfilled shells, we construct a Hund's rule state of eCFs in each layer where we fill orbitals so as to maximize the angular momentum of each layer [57]. Both the $p$-wave and the $s$-wave variational wave functions recover the CF Fermi liquid or Hund's-rule state for a suitable choice of variational parameters, at least when $N$ is such that one has a configuration with either an integral number of completely filled angular-momentum shells, or one with a single CF above the outermost filled shell or with a single CF missing from the outermost shell. At intermediate distances $d / \ell_{B} \sim 1$, the overlaps of both the $p$ - and $s$-wave variational wave functions have dips; however, they remain extremely accurate in this regime. At small interlayer distances, the (111) state is the exact ground state as expected. Both the $p$ wave and the $s$ wave capture this limit as well. As the number of variational parameters is increased, both the $s$-wave and $p$-wave overlaps rapidly improve at small $d$. (In the $s$-wave picture, including more variational parameters allows us to form more tightly bound excitons, hence recovering the (111) state.) Our $s$-wave trial wave functions slightly outperform the previous $p$-wave trial state for an equal number of variational parameters. For example, in Fig. 1(a), both the $p$ - and $s$-wave functions have 5 variational parameters. The squared overlaps at $d=0$ are $0.67,0.95$, and 1.00 for 3,4 , and 5 variational parameters in the $s$-wave case, whereas they are $0.56,0.93$, and 1.00 in the $p$-wave case. (See the Supplemental Material [49] for more details.) Nevertheless, for sufficiently many variational parameters, both the $s$-wave and $p$-wave trial states have extremely good overlaps. It is an interesting open question as to why these seemingly different wave functions appear to describe the same Hilbert space.

Note that the $p$-wave functions described here are putatively the same as those Ref. [26]. However, a detailed comparison will show that the overlaps with exact diagonalization we obtain here are somewhat better, particularly at small $d$, given the same number (or even fewer) variational parameters. In the present work, we use a global optimization algorithm (dual annealing [56]) to optimize the overlaps. Reference [26] used a gradient descent algorithm, which may only find a local optimum of the overlap.

In Fig. 1(b), we use the best variational $s$-wave trial wave function to extract the BCS parameters $\Delta / E_{F}$ and $\xi$ (see the Supplemental Material [49]), where $\xi$ is the coherence length (typical size of a Cooper pair), $\Delta$ is the superconducting order parameter, and $E_{F}$ is the composite fermion Fermi energy. Note that in regular superconductors $\Delta$ is precisely the excitation gap, but here the superconductor has been "composite fermionized," so the excitation gap may not precisely match $\Delta$. We find a crossover from the BEC-like regime $\left(\xi \lesssim \ell_{B}, \Delta \gtrsim E_{F}\right)$ at $d \lesssim \ell_{B}$ to the BCS regime $\left(\xi \gg \ell_{B}, \Delta \ll E_{F}\right)$ at $d \gg \ell_{B}$. In this picture, we have a continuous crossover from the exciton condensate of the (111) state to the BCS-paired composite Fermi liquid.

Charge imbalance.-We now add a charge imbalance to the two layers, while keeping the total filling fraction constant. The filling fractions of the individual layers are 


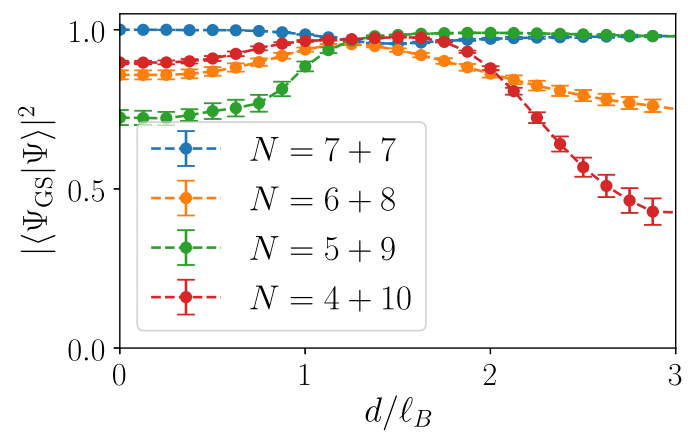

FIG. 2. Exact diagonalization results for the overlap of the $s$-wave BCS trial wave functions with the true ground state for imbalanced layers with a total of 14 electrons (using 6 variational parameters). The $L^{2}=0$ Hilbert space dimensions are 1599 , $1319,614,205$ for $7+7,6+8,5+9$, and $4+10$, respectively. We note that in the limit $d=0$ the Hamiltonian has an enhanced $S U(2)$ pseudospin symmetry, which our trial states do not exhibit, since the overlaps in different pseudospin sectors are not identical.

now $\nu_{\uparrow}=1-\Delta \nu / 2$ and $\nu_{\downarrow}=1+\Delta \nu / 2$. Here, we present results for the charge imbalanced $s$-wave eCF-hCF pairing trial wave functions (see the Supplemental Material for a discussion of the $p$-wave trial wave function for the imbalanced case [49]). In our approach, we compositefermionize the minority carriers in each layer, consistent with the experimental observation that the density of the minority carriers sets the Fermi wave vector away from half-filling $[58,59]$. We show the overlaps of our trial state with the ED ground state in Fig. 2. For small layer imbalances, our trial wave function performs less well at small distances than in the balanced case; however, considering the dimension of the Hilbert space, the high overlaps obtained even in the imbalanced case are significant. At large $d / \ell_{B}$, we expect the layers to form independent $\mathrm{CF}$ states, which will successively fill angular-momentum shells. As mentioned above, our trial wave function Eq. (1) can describe this accurately as long as the shells are either filled, have a single $\mathrm{CF}$ in them, or are one $\mathrm{CF}$ short of being filled. This largely explains why some of the values of $\left(N_{\uparrow}, N_{\downarrow}\right)$ in Fig. 2 are very accurate at large $d / \ell_{B}$ and some are inaccurate in this limit.

Experiments observe enhanced superfluid behavior with layer imbalance [60-62]. We can conjecture the following natural explanation for this. At half-filling, the eCFs (or $\mathrm{hCFs}$ ) are neutral quasiparticles. Away from half-filling, the eCFs in the top layer develop charge $e\left(1-2 \nu_{\uparrow}\right)=e \Delta \nu$, while the hCFs in the bottom layer develop charge $e\left(1-2 \nu_{\downarrow}\right)=-e \Delta \nu$. In the imbalanced case, these two charges can attract to improve the BCS pairing. However, once $\Delta \nu \sim 1 / 2$, we are close to $1 / 4+3 / 4$ and the CF description with two flux quanta attached to each electron or hole should be replaced by a CF description where four flux quanta are attached to each electron or hole. A detailed comparison with experiment [60-62] would require examination of the energies of possible competing phases, which is beyond the scope of this work.

Conclusion.-We proposed a new trial wave function for the bilayer quantum Hall system where eCFs and hCFs pair up in the $s$-wave channel. This trial state has very high overlaps with the exact ground state for any interlayer separation $d$. In this language, the bilayer system undergoes a BEC-BCS crossover as the interlayer separation is varied. At large $d$, the system is in the BCS limit with weakly bound $\mathrm{eCF}-\mathrm{hCF}$ Cooper pairs, whereas at small $d$, the system enters the $\mathrm{BEC}$ regime with tightly bound $\mathrm{eCF}-\mathrm{hCF}$ excitons. We presented numerical results for the BCS order parameter and the coherence length that support this picture. Our trial state also performs extremely well for imbalanced layers and the enhanced stability of the paired state for imbalanced layers seen in experiments can be viewed as a consequence of the CFs developing a nonzero electric charge.

We also reexamined the trial wave function based on pairing eCFs in both layers in the $p$-wave channel and found that, by including sufficiently many variational parameters and by using an improved optimization algorithm compared to Ref. [25], this wave function can also accurately describe the system for any interlayer separation $d$. This is consistent with Ref. [29], which used field theory arguments to show that the $p$-wave state can be continuously connected to the (111) state.

Although both the $s$-wave and the $p$-wave trial states give similar overlaps if sufficiently many variational parameters are included, the BEC-BCS crossover only appears naturally in the $s$-wave picture: $\mathrm{eCF}$ and $\mathrm{hCF}$ excitons are equivalent to the electron and hole excitons forming the (111) state in the tightly bound limit [63]. For the p-wave wave function, there is no simple way to see this (although field theoretic arguments in Ref. [29] suggest that the $p$-wave and (111) state are related).

Numerical calculations were performed using the DiagHam library. We thank Nicolas Regnault for assistance with DiagHam. G. W. would like to thank Gunnar Möller, Ajit Balram, and Frank Pollmann for useful discussions. G. W. thanks the Kavli Institute for Theoretical Physics for its hospitality during the graduate fellowship program. This research was supported in part by the National Science Foundation under Grant No. NSF PHY-1748958 and by the Heising-Simons Foundation. D. X. N. is supported by the Brown Theoretical Physics Center. S. H. S. is supported from EPSRC Grant No. EP/S020527/1. Statement of compliance with EPSRC policy framework on research data: This publication is theoretical work that does not require supporting research data. B. I. H. is supported in part by the Science and Technology Center for Integrated Quantum Materials under NSF Grant No. DMR-1231319. 
[1] J. Eisenstein, Annu. Rev. Condens. Matter Phys. 5, 159 (2014).

[2] J. P. Eisenstein and A. H. MacDonald, Nature (London) 432, 691 (2004).

[3] B. I. Halperin, Helv. Phys. Acta 56, 75 (1983).

[4] B. I. Halperin, P. A. Lee, and N. Read, Phys. Rev. B 47, 7312 (1993).

[5] J. K. Jain, Composite Fermions (Cambridge University Press, Cambridge, England, 2007).

[6] Composite Fermions: A Unified View of the Quantum Hall Regime, edited by O. Heinonen (World Scientific, Singapore, 1998).

[7] K. Moon, H. Mori, K. Yang, S. M. Girvin, A. H. MacDonald, L. Zheng, D. Yoshioka, and S.-C. Zhang, Phys. Rev. B 51, 5138 (1995).

[8] N. E. Bonesteel, I. A. McDonald, and C. Nayak, Phys. Rev. Lett. 77, 3009 (1996).

[9] H. Isobe and L. Fu, Phys. Rev. Lett. 118, 166401 (2017).

[10] T. Morinari, Phys. Rev. B 59, 7320 (1999).

[11] Z. F. Ezawa and G. Tsitsishvili, Rep. Prog. Phys. 72, 086502 (2009).

[12] B. Lian and S.-C. Zhang, Phys. Rev. Lett. 120, 077601 (2018).

[13] Y. N. Joglekar and A. H. MacDonald, Phys. Rev. B 64, 155315 (2001).

[14] Y. N. Joglekar and A. H. MacDonald, Phys. Rev. Lett. 87, 196802 (2001).

[15] Y. N. Joglekar and A. H. MacDonald, Phys. Rev. B 65, 235319 (2002).

[16] A. H. MacDonald, P. M. Platzman, and G. S. Boebinger, Phys. Rev. Lett. 65, 775 (1990).

[17] H. A. Fertig, Phys. Rev. B 40, 1087 (1989).

[18] R. Côté, L. Brey, and A. H. MacDonald, Phys. Rev. B 46, 10239 (1992).

[19] Z. Zhu, L. Fu, and D. N. Sheng, Phys. Rev. Lett. 119, 177601 (2017).

[20] K. Nomura and D. Yoshioka, Phys. Rev. B 66, 153310 (2002).

[21] J. Schliemann, S. M. Girvin, and A. H. MacDonald, Phys. Rev. Lett. 86, 1849 (2001).

[22] N. Shibata and D. Yoshioka, J. Phys. Soc. Jpn. 75, 043712 (2006).

[23] K. Park, Phys. Rev. B 69, 045319 (2004).

[24] K. Park and S. Das Sarma, Phys. Rev. B 74, 035338 (2006).

[25] G. Möller, S. H. Simon, and E. H. Rezayi, Phys. Rev. B 79, 125106 (2009).

[26] G. Möller, S. H. Simon, and E. H. Rezayi, Phys. Rev. Lett. 101, 176803 (2008).

[27] S. H. Simon, E. H. Rezayi, and M. V. Milovanovic, Phys. Rev. Lett. 91, 046803 (2003).

[28] Y.-H. Zhang and I. Kimchi, arXiv:1810.02809.

[29] I. Sodemann, I. Kimchi, C. Wang, and T. Senthil, Phys. Rev. B 95, 085135 (2017).

[30] M. V. Milovanović, E. Dobardžić, and Z. Papić, Phys. Rev. B 92, 195311 (2015).

[31] J. Ye, Phys. Rev. Lett. 97, 236803 (2006).

[32] J. Ye and L. Jiang, Phys. Rev. Lett. 98, 236802 (2007).

[33] J. Alicea, O. I. Motrunich, G. Refael, and M. P. A. Fisher, Phys. Rev. Lett. 103, 256403 (2009).
[34] R. Cipri, Gauge fields and composite fermions in bilayer quantum hall systems, Ph.D. thesis, Florida State University, 2014.

[35] R. Cipri and N. E. Bonesteel, Phys. Rev. B 89, 085109 (2014).

[36] Z. Papic, Fractional quantum Hall effect in multicomponent systems, Ph.D. thesis, Université Paris Sud-Paris XI, 2010.

[37] R. L. Doretto, C. Morais Smith, and A. O. Caldeira, Phys. Rev. B 86, 035326 (2012).

[38] R. L. Doretto, A. O. Caldeira, and C. M. Smith, Phys. Rev. Lett. 97, 186401 (2006).

[39] M. V. Milovanović and S. Djurdjević, Phys. Rev. B 104, 245303 (2021).

[40] B. I. Halperin, Fractional Quantum Hall Effects: New Developments, edited by B. I. Halperin and J. K. Jain (World Scientific, Singapore, 2020), pp. 79-132.

[41] X. Liu, J. I. A. Li, K. Watanabe, T. Taniguchi, J. Hone, B. I. Halperin, P. Kim, and C. R. Dean, arXiv:2012.05916, see in particular Supplementary Material.

[42] D. T. Son, Phys. Rev. X 5, 031027 (2015).

[43] D. X. Nguyen, S. Golkar, M. M. Roberts, and D. T. Son, Phys. Rev. B 97, 195314 (2018).

[44] S. D. Geraedts, M. P. Zaletel, R. S. K. Mong, M. A. Metlitski, A. Vishwanath, and O. I. Motrunich, Science 352, 197 (2016).

[45] E. H. Rezayi and F. D. M. Haldane, Phys. Rev. Lett. 84, 4685 (2000).

[46] M. M. Parish, The BCS-BEC crossover, in Quantum Gas Experiments (Imperial College Press, London, 2014), Chap. 9, pp. 179-197.

[47] Q. Chen, J. Stajic, S. Tan, and K. Levin, Phys. Rep. 412, 1 (2005).

[48] D. X. Nguyen, T. Can, and A. Gromov, Phys. Rev. Lett. 118, 206602 (2017).

[49] See Supplemental Material, which includes Refs. [50-52], at http://link.aps.org/supplemental/10.1103/PhysRevLett .127 .246803 for details of the trial wave functions in the spherical geometry and further numerical results.

[50] T. T. Wu and C. N. Yang, Phys. Rev. D 16, 1018 (1977).

[51] G. Möller and S.H. Simon, Phys. Rev. B 72, 045344 (2005).

[52] Z. Liu, A. C. Balram, Z. Papić, and A. Gromov, Phys. Rev. Lett. 126, 076604 (2021).

[53] The wave function in the lowest Landau level is only a function of $w_{i}^{*}$. Intuitively, one can think of this as arising from the fact the holes have the opposite charge to electrons and thus their cyclotron orbits cycle in the opposite direction in the magnetic field.

[54] J. K. Jain and R. K. Kamilla, Phys. Rev. B 55, R4895 (1997).

[55] R. K. Kamilla, Composite fermions: Physics of 2-dimensional electron systems under strong magnetic fields, Ph.D. thesis, Stony Brook University, 1997.

[56] Y. Xiang, D. Sun, W. Fan, and X. Gong, Phys. Lett. A 233, 216 (1997).

[57] E. Rezayi and N. Read, Phys. Rev. Lett. 72, 900 (1994).

[58] D. Kamburov, Y. Liu, M. A. Mueed, M. Shayegan, L. N. Pfeiffer, K. W. West, and K. W. Baldwin, Phys. Rev. Lett. 113, 196801 (2014). 
[59] M. S. Hossain, M. A. Mueed, M. K. Ma, K. A. Villegas Rosales, Y. J. Chung, L. N. Pfeiffer, K. W. West, K. W. Baldwin, and M. Shayegan, Phys. Rev. Lett. 125, 046601 (2020).

[60] A. R. Champagne, A. D. K. Finck, J. P. Eisenstein, L. N. Pfeiffer, and K. W. West, Phys. Rev. B 78, 205310 (2008).
[61] W. Clarke, A. Micolich, A. Hamilton, M. Simmons, M. Pepper, and D. Ritchie, Physica (Amsterdam) 22E, 40 (2004).

[62] I. B. Spielman, M. Kellogg, J. P. Eisenstein, L. N. Pfeiffer, and K. W. West, Phys. Rev. B 70, 081303(R) (2004).

[63] Note that the phases of the Jastrow factors $\left(z_{i}-z_{j}\right)^{2}\left(w_{i}-\right.$ $\left.w_{j}\right)^{* 2}$ cancel in the tightly bound limit where the eCFs and hCFs are at the same position. 